\title{
COMUNICAÇÃO
}

\section{QUALIDADE FÍSICA E FISIOLÓGICA DE SEMENTES DE BRAQUIÁRIAS COMERCIALIZADAS EM CAMPO GRANDE-MS}

\author{
Physical and physiological quality of seeds of Brachiaria grass commercialized in \\ Campo Grande (Brazil)
}

\author{
Valdemir Antônio Laura', Adriana Paula D’Agostini Contreiras Rodrigues², \\ Edison Rubens Arrabal Arias², Katyuce da Silva Chermouth ${ }^{3}$, Tiago Rossi ${ }^{4}$
}

\begin{abstract}
RESUMO
Conduziu-se, o presente trabalho, no Laboratório Didático de Análise de Sementes da Universidade para o Desenvolvimento do Estado e da Região do Pantanal - UNIDERP, com o objetivo de avaliar a qualidade física e fisiológica de sementes de três espécies de braquiárias (Brachiaria brizantha, B. humidicola e B. decumbens), provenientes de seis empresas estabelecidas no comércio de Campo Grande-MS. Os experimentos foram conduzidos em um delineamento inteiramente casualizado, em esquema fatorial 3 X 6 , com três espécies e seis procedências, totalizando 18 tratamentos. As variáveis analisadas foram: pureza física, primeira contagem do teste de germinação, germinação, valor cultural e massa de 1.000 sementes. Para análise estatística, os dados, quando necessário, foram transformados e as médias comparadas. Há grande variação na qualidade física e fisiológica das sementes de braquiárias comercializadas em Campo Grande-MS; as sementes da espécie B. humidicola, comercializadas em Campo Grande-MS são, em geral, de baixa qualidade física e fisiológica.
\end{abstract}

Termos para indexação: Brachiaria brizantha, Brachiaria humidicola, Brachiaria decumbens, forrageiras, gramíneas.

\section{ABSTRACT}

The present work was carried out at the Didactic Laboratory of Analysis of Seeds of the Universidade para o Desenvolvimento do Estado e da Região do Pantanal - UNIDERP, with the aim of evaluating the physical and physiological quality of seeds of three Brachiaria grass species (Brachiaria brizantha, B. humidicola and B. decumbens), proceeding from six companies located at the commercial area of Campo Grande (Brazil). The experiments were carried out in a completely randomized experimental design in factorial scheme, with four replications. The factors were: species (three) and origins (six); totalizing 18 treatments. The pureness, first counting of the germination test, germination, pure germinating seeds and biomass of 1,000 seeds were evaluated. For the statistical analysis, the data were transformed and the means were compared, when necessary. There is high variation in the physical and physiological quality of the seeds of Brachiaria grass commercialized in Campo Grande. The seeds with best physical and physiological quality of $B$. brizantha specie are from companies 1 and 4 . The seeds of $B$. humidicola, commercialized in Campo Grande presents low physical and physiological quality as a whole. The seeds of $B$. decumbens with the best physical and physiological quality are from companies 1 and 6.

Index terms: Brachiaria brizantha, Brachiaria humidicola, Brachiaria decumbens, forage, grass.

(Recebido em 28 de agosto de 2007 e aprovado em 6 de março de 2008)

O cultivo de pastagens tropicais é relativamente recente no Brasil. A partir da década de 1970, iniciou-se uma expressiva implantação de pastagens, tendo como principal veículo de propagação a semente, em substituição ao restrito método de plantio por mudas. Esse marco histórico foi proporcionado pela semeadura em grande escala das braquiárias em áreas do Cerrado, o que proporcionou um salto nas áreas de pastagens no Brasil, que passaram de 30 milhões para 100 milhões de hectares (ZIMMER \& EUCLIDES, 2000). O Brasil é o maior produtor, consumidor e exportador de sementes forrageiras tropicais (SOUZA, 2002), exportando aproximadamente, 10\% de sua produção anual de sementes de braquiárias (SANTOS FILHO, 1996).

\footnotetext{
'Engenheiro Agrônomo, Doutor, Pesquisador - Embrapa Gado de Corte - Rodovia Br 262, Km 4 - Cx. P. 154 - $79002-970$ - Campo Grande, MS valdemir@cnpgc.embrapa.br

'Engenheiros Agrônomos, Doutores, Professores - Universidade para o Desenvolvimento do Estado e da Região do Pantanal/UNIDERP Rua Alexandre Herculano, 1400 - Jardim Veraneio - Cx. P. 2153 - 79037-280 - Campo Grande, MS - adricontreiras@hotmail.com, edisonarias@mail.uniderp.br

${ }^{3}$ Engenheira Agrônoma - Estância Jóia, Km 6 - Estrada São Julião - 79000-000 - Campo Grande, MS - katychermouth@yahoo.com.br

${ }^{4}$ Engenheiro Agrônomo - Rua Antônio Pereira dos Santos, 569 - Vila Gisele - 79990-000 - Amambaí, MS - tiago_rossi1984@yahoo.com.br
} 
A produção de sementes é um processo complexo e condicionado por uma série de fatores específicos; entre as principais características, estão o período prolongado da emissão das inflorescências e da abertura de flores entre e dentro de uma mesma inflorescência, queda natural (degrana) das sementes, por ocasião da maturidade, ou mesmo antes, dormência das sementes (BOONMAM, 1971) e o sistema de colheita e de beneficiamento de sementes, o que, em geral, ocasiona grande diferença na qualidade física e fisiológica dessas sementes. As informações sobre a adequada avaliação da qualidade fisiológica das sementes dessa forrageira, a mais cultivada no Brasil, ainda não são satisfatórias (LAGO \& MARTINS, 1998).

As pastagens cultivadas são a base da produção da pecuária bovina de corte no Brasil. O gênero Brachiaria agrupa as cultivares de gramíneas forrageiras mais importantes para a produção de carne bovina. A região Centro-Oeste do Brasil comporta, na atualidade, aproximadamente, $30 \%$ do rebanho bovino nacional, com áreas de pastagens em torno de 60 milhões de hectares. As espécies do gênero Brachiaria passaram a ter uma grande importância para a pecuária brasileira a partir da década de 1970, por ocuparem grandes extensões territoriais, sobretudo na região dos Cerrados. Estima-se que o Brasil tenha mais de 120 milhões de hectares de pastagens cultivadas, e que mais de $85 \%$ da área seja ocupada por braquiárias (BARBOSA, 2006).

Em sua essência, a semente transporta a garantia da perpetuação de cada espécie cultivada. Existe um grande número de fatores que afetam a qualidade das sementes, destacando-se os genéticos, fisiológicos e ambientais. Os fisiológicos têm sua ação determinada pelo ambiente durante a produção, a colheita, beneficiamento e armazenamento.

A massa de sementes de gramíneas forrageiras pode variar entre os anos, causada possivelmente, por variações climáticas (ANDRADE, 1983). Também a adubação nitrogenada, época de colheita e manejo são outros fatores que influenciam nessa variação (CONDÉ, 1982). Como a viabilidade de sementes geralmente apresenta seus maiores valores na maturidade fisiológica, que por sua vez, é definida pela massa máxima que a semente atinge no seu processo de maturação, a massa de 1.000 sementes é um parâmetro que pode ser utilizado para estimar a qualidade fisiológica (vigor) de lotes de sementes.

Objetivou-se, no presente trabalho, avaliar a qualidade física e fisiológica de sementes de três espécies de braquiárias (Brachiaria brizantha, B. humidicola e $B$. decumbens), provenientes de seis empresas estabelecidas no comércio de Campo Grande-MS.
O presente trabalho foi conduzido no Laboratório Didático de Análise de Sementes da Universidade para o Desenvolvimento do Estado e da Região do Pantanal UNIDERP, em Campo Grande-MS, de 20/11/2006 a 28/02/ 2007.

As sementes de três espécies de braquiárias (Brachiaria brizantha, B. humidicola e B. decumbens), safra 2005/2006, procedentes de seis empresas foram adquiridas no comércio de Campo Grande-MS. Os experimentos foram conduzidos em um delineamento inteiramente casualizado, em esquema fatorial 3 X 6, com três espécies e seis procedências, totalizando 18 tratamentos.

As variáveis analisadas foram:

Pureza (\%): determinada segundo as Regras para Análise de Sementes (BRASIL, 1992).

As demais avaliações foram realizadas a partir da porção de sementes puras obtidas nesse teste.

Primeira contagem (\%): realizada aos sete dias após o início do teste padrão de germinação, com quatro repetições de 100 sementes.

Germinação $(\%)$ : realizada com quatro repetições de 100 sementes, conforme as Regras para Análise de Sementes - RAS (BRASIL, 1992), ou seja, o teste foi conduzido sobre papel umedecido de acordo com o peso do papel vezes 2,5 , e colocado para germinar na temperatura de $20-35^{\circ} \mathrm{C}$, com fotoperíodo de 12 horas, sendo que as contagens foram realizadas aos sete e 21 dias. Não foram realizados tratamentos para superação da dormência.

Valor cultural (\%): realizada com quatro repetições de 100 sementes, conforme as Regras para Análise de Sementes (BRASIL, 1992).

Massa de 1.000 sementes (g): obtida pela avaliação da massa de oito repetições de 100 sementes, segundo as determinações das Regras para Análise de Sementes (BRASIL, 1992).

Para análise estatística, os dados, quando necessário, foram transformados conforme preconizado por Santana \& Ranal (2004) e as médias comparadas pelo teste de Tukey, a 5\% de probabilidade. A variável pureza não foi analisada estatisticamente, mas de acordo com o que estabelece as RAS (BRASIL, 1992).

Numericamente, a maior porcentagem de pureza das sementes de braquiária foi obtida para a espécie Brachiaria brizantha proveniente da empresa 4 , seguida da mesma espécie para a empresa 2 (Tabela 1). Para a espécie $B$. humidicola, a maior porcentagem de pureza foi obtida em sementes provenientes da empresa 1 , seguida da mesma espécie para as empresas 6 e 4, indicando que as essas 
empresas são, entre as seis empresas avaliadas, as que comercializam sementes com maior pureza física. Meschede et al. (2004) avaliando três lotes de sementes de $B$. brizantha encontraram valores de pureza física que variaram entre 28 e $41 \%$, valores esses inferiores às piores procedências dessa espécie observados no presente trabalho, evidenciando um aumento da qualidade física das sementes que estão sendo produzidas, decorrente da profissionalização dos produtores de sementes.

Para sementes de $B$. decumbens, observou-se que não houve grande variação na porcentagem de pureza, independente da procedência, sendo de maneira geral, a espécie com menor pureza.

Com relação à primeira contagem do teste de germinação de sementes de braquiária (Tabela 2), os maiores valores foram verificados para $B$. decumbens procedentes da empresa 6, que não diferiu dos resultados obtidos com sementes da empresa 1 e resultados intermediários foram obtidos com sementes da empresa 4. Para as sementes de B. brizantha, houve uma grande variação nos resultados obtidos de acordo com cada procedência, destacandose as amostras das empresas 1 e 4. De uma maneira geral, as menores porcentagens de germinação, na primeira contagem, foram registradas para a espécie $B$. humidicola.

$\mathrm{Na}$ Tabela 3, apresentam-se os resultados da porcentagem de germinação de sementes de braquiária, que provavelmente não foram influenciados pela dormência, visto que essas sementes foram colhidas entre fevereiro e junho do ano de 2006, tendo tempo suficiente para superação da dormência, e já estavam disponíveis no comércio (no momento em que foram adquiridas para o

Tabela 1 - Porcentagem de pureza de sementes de Brachiaria brizantha, B. humidicola e B. decumbens, provenientes de seis empresas estabelecidas no comércio de Campo Grande-MS.

\begin{tabular}{|c|c|c|c|c|}
\hline \multirow{2}{*}{ Procedências } & \multicolumn{3}{|c|}{ Espécies } & \multirow[t]{2}{*}{ Médias } \\
\hline & B. brizantha & B. humidicola & B. decumbens & \\
\hline 1 & 91,56 & 73,75 & 56,12 & 73,81 \\
\hline 2 & 53,88 & 45,19 & 46,97 & 48,68 \\
\hline 3 & 43,58 & 41,82 & 39,40 & 41,60 \\
\hline 4 & 93,79 & 61,18 & 44,57 & 66,51 \\
\hline 5 & 44,91 & 58,50 & 54,82 & 52,74 \\
\hline 6 & 54,87 & 61,65 & 39,55 & 52,02 \\
\hline Média & 63,77 & 57,01 & 46,90 & \\
\hline Desvio padrão & 22,86 & 11,76 & 7,26 & \\
\hline
\end{tabular}

Tabela 2 - Primeira contagem do teste de germinação de sementes de Brachiaria brizantha, B. humidicola e B. decumbens, provenientes de seis empresas estabelecidas no comércio de Campo Grande-MS.

\begin{tabular}{|c|c|c|c|c|c|}
\hline \multirow{3}{*}{$\begin{array}{c}\text { Procedências } \\
1\end{array}$} & \multicolumn{5}{|c|}{ Espécies } \\
\hline & B. brizantha & \multicolumn{2}{|c|}{ B. humidicola } & \multicolumn{2}{|c|}{ B. decumbens } \\
\hline & $27 \mathrm{AB}$ & $3 \mathrm{AB}$ & \multirow{2}{*}{$\begin{array}{l}\mathrm{c} \\
\mathrm{c}\end{array}$} & $44 \mathrm{AB}$ & $\mathrm{a}$ \\
\hline 2 & $10 \quad \mathrm{C} \quad \mathrm{b}$ & $2 \mathrm{~B}$ & & $24 \mathrm{C}$ & $\mathrm{a}$ \\
\hline 3 & $22 \quad \mathrm{~B} \quad \mathrm{a}$ & $4 \mathrm{AB}$ & $\mathrm{b}$ & $24 \quad \mathrm{C}$ & $\mathrm{a}$ \\
\hline 4 & $39 \mathrm{~A}$ & $2 \mathrm{~B}$ & $\mathrm{~b}$ & $41 \quad \mathrm{~B}$ & $\mathrm{a}$ \\
\hline 5 & $1 \quad \mathrm{Dab}$ & $1 \mathrm{~B}$ & $\mathrm{~b}$ & $22 \mathrm{C}$ & $\mathrm{a}$ \\
\hline 6 & $4 \quad \mathrm{CDa}$ & $11 \mathrm{~A}$ & $\mathrm{a}$ & $56 \mathrm{~A}$ & $\mathrm{a}$ \\
\hline $\mathrm{CV}$ & \multicolumn{3}{|c|}{$16,98 \%$} & & \\
\hline
\end{tabular}

Médias seguidas pela mesma letra minúscula na linha e maiúscula na coluna não diferem entre si (Tukey 1\%). Médias originais, para análise estatística e comparação das médias, os dados foram transformados em arco.seno $\frac{\sqrt{\mathrm{x}+0,5}}{100}$. 
experimento), teoricamente aptas para imediata semeadura em campo, portanto, não se justificou a utilização de tratamento para superação da dormência, uma vez que nenhum produtor o faria.

Pôde-se constatar (Tabela 3 ) que a espécie $B$. brizantha proveniente da empresa 4 foi a que apresentou os maiores valores de germinação, não diferindo dos resultados obtidos com sementes provenientes da empresa 1, que também foram as que apresentaram maior porcentagem de pureza (Tabela 1).

Dias \& Toledo (1993), avaliando a germinação de sementes de $B$. brizantha obtiveram valores entre 51 e 55 $\%$ e Novembre et al. (2006), observaram valores entre 77 e $90 \%$. Entretanto, Meschede et al. (2004) avaliando três lotes de sementes de $B$. brizantha, verificaram uma variação na porcentagem de germinação de 27 a 64 \%, evidenciando, assim como no presente trabalho, uma heterogeneidade entre as amostras coletadas de diferentes procedências.

Para a espécie $B$. decumbens, os maiores valores de germinação foram registrados para sementes provenientes da empresa 6 , embora não tenha diferido dos resultados obtidos nas amostras das empresas 1, 3 e 4. Já para $B$. humidicola não houve diferenças significativas entre as amostras e os valores observados foram muito inferiores às demais espécies, independente da procedência e, também, inferiores aos valores encontrados por Macedo et al. (1994).

De uma maneira geral, as sementes de gramíneas forrageiras são comercializadas com base no valor cultural (VC), que exprime a porcentagem de germinação e a pureza em uma única variável, sendo que, no Brasil, o VC médio das sementes de forrageiras comercializadas está em torno de 30\%. Para as espécies e procedências avaliadas, o maior $\mathrm{VC}$ foi obtido para as sementes da espécie $B$. brizantha provenientes das empresas 4 e 1, respectivamente (Tabela 4), estando acima dos valores médios das sementes comercializadas, o que denota maior qualidade dessas sementes. As sementes de B. humidicola apresentaram, em comparação com as demais, os mais baixos VC, sendo que o maior $\mathrm{VC}$ foi observado para as sementes da empresa 6 , embora não tenha diferido das sementes comercializadas pela empresa 1, abaixo ainda, dos valores médios de mercado. $\mathrm{O}$ mais alto VC para sementes de $B$. decumbens foi registrado para a empresa 1, seguido das empresas 6 e 4.

A massa das sementes é influenciada pelas condições climáticas, região geográfica, época de colheita, nutrição das plantas e, principalmente, pelas características genéticas de cada espécie. Numericamente, as sementes de maior massa foram as de B. brizantha, seguidas por $B$. decumbens e as de menor massa foram as de $B$. humidicola (Tabela 5). A empresa 1 foi a que apresentou as sementes de $B$. brizantha e $B$. humidicola de maior massa, enquanto que as sementes de $B$. decumbens com maior massa foram aquelas provenientes da empresa 6.

Observou-se também que houve uma relação direta entre as variáveis na primeira contagem do teste de germinação, porcentagem de germinação e massa de 1.000 sementes de $B$. brizantha, o que também foi registrado por Meschede et al. (2004) para essa mesma espécie. Os maiores valores, para essas variáveis, foram obtidos por sementes provenientes da empresa 1. A mesma relação foi constatada para sementes de B. decumbens, provenientes da empresa 6 . Já para $B$. humidicola não foi evidenciada nenhuma relação entre as variáveis.

Tabela 3 - Porcentagem de germinação de sementes de Brachiaria brizantha, B. humidicola e B. decumbens, provenientes de seis empresas estabelecidas no comércio de Campo Grande-MS.

\begin{tabular}{|c|c|c|c|c|c|c|}
\hline \multirow{3}{*}{$\begin{array}{c}\text { Procedências } \\
1\end{array}$} & \multicolumn{6}{|c|}{ Espécies } \\
\hline & \multicolumn{2}{|c|}{ B. brizantha } & \multicolumn{2}{|c|}{ B. humidicola } & \multicolumn{2}{|c|}{ B. decumbens } \\
\hline & $59 \mathrm{~A}$ & $\mathrm{a}$ & $9 \mathrm{~A}$ & $\mathrm{~b}$ & $50 \mathrm{AB}$ & $\mathrm{a}$ \\
\hline 2 & $18 \mathrm{BC}$ & $\mathrm{a}$ & $4 \mathrm{~A}$ & $\mathrm{~b}$ & $27 \mathrm{BC}$ & $\mathrm{a}$ \\
\hline 3 & $29 \mathrm{~B}$ & $\mathrm{a}$ & $7 \mathrm{~A}$ & $\mathrm{~b}$ & $43 \mathrm{ABC}$ & $\mathrm{a}$ \\
\hline 4 & $67 \mathrm{~A}$ & $\mathrm{a}$ & $4 \mathrm{~A}$ & $\mathrm{c}$ & $46 \mathrm{ABC}$ & $a b$ \\
\hline 5 & 1 & $b$ & $3 \mathrm{~A}$ & $\mathrm{~b}$ & 25 & $\mathrm{a}$ \\
\hline 6 & 6 & $b$ & $15 \mathrm{~A}$ & $\mathrm{~b}$ & $58 \mathrm{~A}$ & $\mathrm{a}$ \\
\hline
\end{tabular}

Médias seguidas pela mesma letra minúscula na linha e maiúscula na coluna não diferem entre si (Tukey 1\%). Médias originais, para análise estatística e comparação das médias, os dados foram transformados em arco.seno $\frac{\sqrt{x+0,5}}{100}$. 
Tabela 4 - Valor cultural de sementes de Brachiaria brizantha, B. humidicola e B. decumbens, provenientes de seis empresas estabelecidas no comércio de Campo Grande-MS.

\begin{tabular}{|c|c|c|c|}
\hline \multirow{3}{*}{$\begin{array}{c}\text { Procedências } \\
1\end{array}$} & \multicolumn{3}{|c|}{ Espécies } \\
\hline & B. brizantha & B. humidicola & B. decumbens \\
\hline & $54,0 \mathrm{~A} \quad \mathrm{a}$ & $6,5 \mathrm{AB} \quad \mathrm{c}$ & $27,8 \mathrm{~A} \quad \mathrm{~b}$ \\
\hline 2 & $9,7 \quad \mathrm{~B} \quad \mathrm{a}$ & $1,6 \quad \mathrm{BC} \mathrm{b}$ & $12,7 \quad \mathrm{~B} \quad \mathrm{a}$ \\
\hline 3 & $12,5 \mathrm{~B} \quad \mathrm{a}$ & $2,9 \quad \mathrm{BC} b$ & 16,9 B a \\
\hline 4 & $62,8 \mathrm{~A} \quad \mathrm{a}$ & $2,1 \quad \mathrm{BC} \quad \mathrm{c}$ & $20,5 \mathrm{AB} \quad \mathrm{b}$ \\
\hline 5 & $0,4 \quad \mathrm{C} \mathrm{b}$ & $1,6 \quad \mathrm{C} \mathrm{b}$ & $13,5 \quad \mathrm{~B}$ a \\
\hline 6 & $3,3 \quad \mathrm{C} \quad \mathrm{c}$ & $9,1 \mathrm{~A} \quad \mathrm{~b}$ & $22,7 \mathrm{AB} \quad \mathrm{a}$ \\
\hline Média & 23,7 & 4,0 & 19,0 \\
\hline $\mathrm{CV}$ & & $16,85 \%$ & \\
\hline
\end{tabular}

Médias seguidas pela mesma letra minúscula na linha e maiúscula na coluna não diferem entre si (Tukey 1\%). Médias originais, para análise estatística e comparação das médias, os dados foram transformados em arco.seno $\frac{\sqrt{x+0,5}}{100}$.

Tabela 5 - Massa de mil sementes de Brachiaria brizantha, B. humidicola e $B$. decumbens, provenientes de seis empresas estabelecidas no comércio de Campo Grande-MS.

\begin{tabular}{|c|c|c|c|c|c|c|c|}
\hline \multirow{3}{*}{$\begin{array}{c}\text { Procedências } \\
1\end{array}$} & \multicolumn{7}{|c|}{ Espécies } \\
\hline & \multicolumn{2}{|c|}{ B. brizantha } & \multicolumn{3}{|c|}{ B. humidicola } & \multicolumn{2}{|c|}{ B. decumbens } \\
\hline & \multicolumn{2}{|c|}{$9,5675 \mathrm{~A}$} & \multicolumn{2}{|c|}{$4,1475 \mathrm{~A}$} & \multirow{2}{*}{$\begin{array}{l}\mathrm{c} \\
\mathrm{c}\end{array}$} & 5,4625 & $\mathrm{C}$ \\
\hline 2 & 7,4675 & $\mathrm{E}$ & 3,4725 & $\mathrm{C}$ & & 5,6325 & B \\
\hline 3 & 8,4400 & $\mathrm{D}$ & 3,8525 & B & $\mathrm{c}$ & 5,3725 & $\mathrm{C}$ \\
\hline 4 & 9,1025 & $\mathrm{~B}$ & 3,7900 & B & $\mathrm{c}$ & 5,6250 & B \\
\hline 5 & 7,3850 & $\mathrm{E}$ & 3,8225 & B & $\mathrm{c}$ & 4,8225 & $\mathrm{D}$ \\
\hline 6 & 8,6425 & $\mathrm{C}$ & 3,8400 & $\mathrm{~B}$ & $\mathrm{c}$ & $5,8000 \mathrm{~A}$ & \\
\hline Média & 8,4 & 42 & & 8208 & & 5,45 & \\
\hline DMS & 0,1 & 17 & & 0847 & & 0,11 & \\
\hline $\mathrm{CV}$ & 0,5 & $\%$ & & $99 \%$ & & 0,90 & \\
\hline
\end{tabular}

Médias seguidas pela mesma letra maiúscula na coluna não diferem entre si (Tukey 1\%).

Nas condições experimentais avaliadas, pôde-se concluir que há grande variação na qualidade física e fisiológica das sementes de braquiárias comercializadas em Campo Grande (MS) e que, as sementes de $B$. humidicola, comercializadas em Campo Grande (MS) são, em geral, de baixa qualidade física e fisiológica.

\section{REFERÊNCIAS BIBLIOGRÁFICAS}

ANDRADE, R. V. Épocas de colheita, produção e qualidade de sementes de capim gordura. Revista Brasileira de Sementes, Brasília, v. 2, n. 5, p. 9-22, 1983.

BARBOSA, R. A. Morte de pastos de braquiárias. Campo Grande: Embrapa Gado de Corte, 2006. 206 p.
BOONMAN, J. G. Experimental studies on seed production of tropical grasses in Kenya-1: general introduction and analysis of problems. Netherlands Journal of Agricultural Science, [S.1.], v. 19, p. 23-36, 1971.

BRASIL. Ministério da Agricultura e Reforma Agrária. Regras para análise de sementes. Brasília, DF: SNDA/ DNDV/CLAV, 1992. 365 p.

CONDÉ, A. dos R. Produção de forrageiras no cerrado. In: SIMPÓSIO NACIONAL SOBRE SEMENTES DE FORRAGEIRAS, 2., 1982, Nova Odessa. Anais... São Paulo: [s.n.], 1982. p. 51-66. 
DIAS, D. C. F. S.; TOLEDO, F. F. de. Germinação e incidência de fungos em testes com sementes de Brachiaria brizantha Stapf. Scientia Agrícola, Piracicaba, v. 50, n. 1, p. 68-73, 1993.

LAGO, A. A.; MARTINS, L. Qualidade fisiológica de sementes de Brachiaria brizantha. Pesquisa Agropecuária Brasileira, Brasília, v. 33, n. 2, p. 199-204, 1998.

MACEDO, E. C.; GROTH, D.; LAGO, A. A. do. Efeito de escarificação com ácido sulfúrico na germinação de sementes de Brachiaria humidicola (Rendle) Schweick. Pesquisa Agropecuária Brasileira, Brasília, v. 29, n. 3, p. 455-460, 1994.

MESCHEDE, D. K.; SALES, J. G. C.; BRACCINI, A. de L.; SCAPIM, C. A.; SCHUAB, S. R. P. Tratamentos para superação da dormência das sementes de capim-braquiária cultivar Marandu. Revista Brasileira de Sementes, Brasília, v. 26, n. 2, p. 76-81, 2004.

NOVEMBRE, A. D. da L. C.; CHAMMA, H. M. C. P.; GOMES, R. B. R. Viabilidade das sementes de braquiária pelo teste de tetrazólio. Revista Brasileira de Sementes, Brasília, v. 28, n. 2, p. 147-151, 2006.

SANTANA, D. G.; RANAL, M. A. Análise da germinação: um enfoque estatístico. Brasília, DF: UnB, 2004. 248 p.

SANTOS FILHO, L. F. Seed production: perspective from the brazilian private sector. In: MILES, J. W.; MAASS, B. L.; VALLE, C. B. (Eds.). Brachiaria: biology, agronomy, and improvement. Cali: CIAT, 1996. p. 141-146.

SOUZA, F. H. D. de; ANDRADE, R. P. de. Qualidade e preparo de sementes forrageiras In: Curso formação de pastagens. Campo Grande: Embrapa Gado de Corte, 2002. Não paginado.

ZIMMER, A. H.; EUCLIDES, V. P. B. Importância das pastagens para o futuro da pecuária de corte no Brasil. In: SIMPÓSIO DE FORRAGICULTURA E PASTAGENS, 1., 2000, Lavras. Temas em Evidências. Lavras: UFLA, 2000. p. $1-50$. 